\title{
Erratum to: Prevalence of patent foramen ovale in ischemic stroke in Italy: the SISIFO Study
}

\author{
Domenico Consoli $^{1}$ • Maurizio Paciaroni ${ }^{3} \cdot$ Marco Aguggia $^{4} \cdot$ Maurizio Melis $^{5}$ • \\ Giovanni Malferrari ${ }^{6}$ - Simone Vidale $^{7} \cdot$ Paolo Cerrato $^{2} \cdot$ Simona Sacco $^{8}$. \\ Carlo Gandolfo $^{9}$ - Paolo Bovi ${ }^{10}$ - Carlo Serrati ${ }^{11}$ - Massimo Del Sette ${ }^{12}$. \\ Anna Cavallini $^{13} \cdot$ Marina Diomedi $^{14} \cdot$ Paolo Postorino $^{1} \cdot$ Stefano Ricci $^{15}$
}

Published online: 21 April 2015

(C) Springer-Verlag Italia 2015

\section{Erratum to: Neurol Sci (2014) 35:867-873 DOI 10.1007/s10072-013-1615-2}

The affiliation details of the co-authors were incorrect in the original publication of the article. The correct information is given below:

P. Cerrato

AOU "Molinette", Turin, Italy

C. Serrati

AO "S. Martino", Genoa, Italy
M. Del Sette

Neurology Unit, S. Andrea Hospital, Via Vittorio Veneto 197, 19124 La Spezia, Italy

A. Cavallini

UC Malattie Cerebrovascolari/Stroke Unit, IRCCS "C. Mondino", Pavia, Italy

M. Diomedi

UOC Stroke Unit, Dipartimento di Neuroscienze, Università degli Studi di Roma Tor Vergata, Rome, Italy

S. Ricci

UO Neurologia, ASL 1 dell'Umbria Ospedale, Via Engels, 06012 Città di Castello (PG), Italy

9 DINOGMI, Stroke-Unit, University of Genoa, Largo Daneo, 3, 16132 Genoa, Italy

10 SSO Stroke Unit UO Neurologia DAI di Neuroscienze, AOU Integrata Verona, Verona, Italy

11 AO "S. Martino", Genoa, Italy

12 Neurology Unit, S. Andrea Hospital, Via Vittorio Veneto 197, 19124 La Spezia, Italy

13 UC Malattie Cerebrovascolari/Stroke Unit, IRCCS “C. Mondino”, Pavia, Italy

14 UOC Stroke Unit, Dipartimento di Neuroscienze, Universita' degli Studi di Roma Tor Vergata, Rome, Italy

15 UO Neurologia ASL 1 dell' Umbria Ospedale, Via Engels, 06012 Città di Castello (PG), Italy Reggio Emilia, Italy

7 Azienda Ospedaliera S. Anna, Como, Italy

8 Department of Neurology, University of L'Aquila, 67100 L'Aquila, Italy 